\title{
BMJ Open Acute kidney injury and 1-year mortality after colorectal cancer surgery: a population-based cohort study
}

\author{
Charlotte Slagelse, ${ }^{\oplus 1}$ Henrik Gammelager, ${ }^{1,2}$ Lene Hjerrild Iversen, ${ }^{3}$ \\ Henrik Toft Sørensen, ${ }^{1}$ Christian F Christiansen ${ }^{1}$
}

To cite: Slagelse C, Gammelager H, Iversen LH, et al. Acute kidney injury and 1-year mortality after colorectal cancer surgery: a populationbased cohort study. BMJ Open 2019;9:e024817. doi:10.1136/ bmjopen-2018-024817

- Prepublication history and additional material for this paper are available online. To view these files, please visit the journal online (http://dx.doi. org/10.1136/bmjopen-2018024817).

Received 15 June 2018 Revised 26 December 2018 Accepted 22 January 2019

Check for updates

(c) Author(s) (or their employer(s)) 2019. Re-use permitted under CC BY-NC. No commercial re-use. See rights and permissions. Published by BMJ.

${ }^{1}$ Department of Clinical Epidemiology, Aarhus University Hospital, Aarhus N, Denmark ${ }^{2}$ Department of Intensive Care, Aarhus University Hospital, Aarhus N, Denmark

${ }^{3}$ Department of Surgery, Aarhus University Hospital, Aarhus N, Denmark

Correspondence to Dr Charlotte Slagelse; csjh@clin.au.dk

\section{ABSTRACT}

Objectives Acute kidney injury (AKI) is a frequent postoperative complication, but the mortality impact within different postoperative time frames and severities of AKI are poorly understood. We examined the occurrence of postoperative AKI among colorectal cancer (CRC) surgery patients and the impact of AKI on mortality during 1 year after surgery.

Design Observational cohort study. We defined the exposure, AKI, as a $50 \%$ increase in plasma creatinine or initiation of renal replacement therapy within 7 days after surgery or an absolute increase in creatinine of $26 \mu \mathrm{mol} / \mathrm{L}$ within 48 hours.

Setting Population-based Danish medical databases. Participants A total of 6580 patients undergoing CRC surgery in Northern Denmark during 2005-2011 were included from the Danish Colorectal Cancer Group database.

Outcomes measure Occurrence of AKI and 8-30, 31-90 and $91-365$ days mortality in patient with or without AKI. Results AKI occurred in 1337 patients (20.3\%) of the 6580 patients who underwent CRC surgery. Among patients with AKI, 8-30, 31-90 and 91-365 days mortality rates were $10.1 \%(95 \% \mathrm{Cl} 8.6 \%$ to $11.9 \%), 7.8 \%(95 \%$ Cl $6.4 \%$ to $9.5 \%)$ and $12.0 \%(95 \% \mathrm{Cl} 10.3 \%$ to $14.2 \%)$, respectively. Compared with patients without AKI, AKI was associated with increased 8-30 days mortality (adjusted $\mathrm{HR}(\mathrm{aHR})=4.01,95 \% \mathrm{Cl} 3.11$ to 5.17$)$ and 3190 days mortality (aHR $2.08,95 \% \mathrm{Cl} 1.60$ to 2.69 ), while 91-365 days aHR was $1.12(95 \% \mathrm{Cl} 0.89$ to 1.41$)$. We observed no major differences in stratified analyses. Conclusions AKI after surgery for CRC is a frequent postoperative complication associated with a substantially increased 90-day mortality. AKI should be considered a potential target for reducing 90-day mortality.

\section{INTRODUCTION}

Colorectal cancer (CRC) is the third most common cancer in the world with an annual incidence of 1 in $360000 .{ }^{1}$ Surgery plays a crucial role in the treatment of CRC. As the median age is above 70 years at the time of CRC diagnosis, CRC surgery is often performed in elderly patients with comorbidities. Thirty-day mortality ${ }^{2-5}$ after CRC surgery has been reported to range from $0.4 \%$ to $23 \%$ and $7 \%$

\section{Strengths and limitations of this study}

No former studies examined the impact of acute kidney injury (AKI) (and stages) on 1-year mortality after colorectal cancer surgery.

- We used Danish population-based administrative medical and clinical quality databases.

- Participants had uniform access to healthcare and virtually complete follow-up.

- We defined AKI according to current guidelines Kidney Disease Improving Global Outcomes.

- Urine output was unavailable which may have led to a minor underestimation of AKI.

to $65 \%$ of CRC surgery patients experience postoperative complications ${ }^{26}$ within 30 days after the surgery. The wide range of complication and mortality rates could be explained by the fact that the studies are heterogeneous as regards the included procedures and the urgency of surgery.

Acute kidney injury (AKI) is a common postoperative complication $^{7}$ defined by a sudden decline in the excretory function of the kidneys, ranging from mild subclinical AKI to AKI patients receiving renal replacement therapy (RRT) ${ }^{89}$ In a Danish population-based study of mortality after emergency surgery for colon cancer, mortality during the first 30 days after surgery was increased in patients with decreased kidney function (receiving RRT). ${ }^{10}$ A small cohort study included 288 medical records from elective rectal cancer surgeries and found an AKI occurrence of $3.8 \%$ and an in-hospital mortality of $18.2 \%$ in patients with AKI, whereas, patients without AKI had an in-hospital mortality of $0.7 \% .^{11}$ Studies of other major abdominal surgery reported postoperative AKI in 3\%-35\% of patients, ${ }^{7}$ and found AKI to be associated with increased mortality ${ }^{2}$ However, no previous study has examined the impact of AKI severity on mortality within different time frames during a year after CRC surgery. The results of the current study, 
therefore, intend to provide results for designing future well-targeted interventions aiming at reducing postoperative AKI and the associated mortality. Knowledge about occurrence and prognosis of postoperative AKI in patients undergoing CRC surgery could help determine whether these are high-risk patients requiring special attention after surgery. Therefore, we examined the occurrence of AKI and its prognostic impact on mortality within 1 year after CRC surgery.

\section{MATERIALS AND METHODS \\ Study design and setting}

This cohort study was conducted in Northern Denmark (the North and Central Denmark Regions, with 2074956 cumulative inhabitants during the study period) using prospectively collected data from medical databases. ${ }^{12-16}$ Tax-supported healthcare is provided by the Danish National Health Service to all Danish residents. Since 1968, all residents have been assigned a unique 10-digit civil registration number (CPR number in Danish), which allows unambiguous individual-level linkage between public databases. ${ }^{14}$ In the current study, CRC surgeries were performed at nine hospitals in the study area.

\section{Patient and public involvement}

Patients and public were not involved in the development or the design of this study.

\section{Study population}

This study included all patients who underwent first time CRC surgery during January 2005 to December 2011. Patients were identified in the Danish Colorectal Cancer Group (DCCG.dk) database. ${ }^{16}$ This database, established in May 2001, contains information on patients with CRC covering demographics, treatments, complications including occurrence of patients with AKI receiving RRT and mortality within 30 days after surgery. To ensure availability of baseline laboratory data, we required residency in the study area in a period with laboratory data available. ${ }^{12}$ Moreover, patients with chronic dialysis within 30 days before surgery and patients who died within 7 days after surgery were excluded from the study.

\section{Acute kidney injury}

Data on plasma creatinine (PCr) (equivalent to serum creatinine $[\mathrm{SCr}]$ ) were retrieved from the clinical laboratory information system (LABKA) research database. ${ }^{12} 17$ This database contains PCr test results collected by general practices and hospitals in the study area since 1990. We defined AKI as a $50 \%$ increase in PCr, initiation of RRT within 7 days after surgery or an absolute increase in creatinine of $26 \mu \mathrm{mol} / \mathrm{L}$ within 48 hours. We also identified the highest AKI stage occurring within 7 days after CRC surgery according to the SCr criteria in the recent Kidney Disease Improving Global Outcomes (KDIGO) consensus criteria, as follows: no AKI, stage 1 AKI, stage 2 AKI and stage 3 AKI (figure 1). ${ }^{8}$ Further, we calculated mean durations of AKI (in days) defined as the number of days with AKI without a normal creatinine and with the requirement of no more than one 1 day between creatinine samples. In our main analysis, we included patients without a PCr measurement within 7 days following surgery (ie, lacking exposure information) in the group of patients without AKI, based on two rationales. First, a blood draw is minimally invasive and low cost; hence, the indication threshold is low. Second, the hospitals that contributed the most patients without exposure information followed a fast-track protocol. ${ }^{23}$ This protocol advises physicians and nurses to refrain from postoperative blood analyses if a patient is healthy and recovering well. Hence, we expected patients without exposure information to resemble healthy patients without AKI. To examine this assumption, we created a second version of table 1 , putting patients with missing AKI data in a separate category. Patients lacking one or more PCr measurements within 7 days following surgery $(10.9 \%, \mathrm{n}=602)$ appeared healthier than patients with AKI and, in general, resembled patients without AKI.

\section{Mortality}

We obtained data on mortality, from day 8 after CRC surgery and until 1 year after this surgery, from the Danish Civil Registration System (CRS). The CRS has maintained complete information on all changes in vital status and migration for the entire Danish population since $1968 .^{14}$

\section{Covariates}

Preoperative covariates were chosen based on their potential association with AKI and with postoperative mortality. $^{7-9} 18$ The Danish National Patient Registry (DNPR) contains information on all hospitalisations since 1977, outpatient visits since 1994 and emergency room visits since 1995 . The DNPR includes information on diagnoses, procedures and admission/discharge dates. Chronic kidney disease (CKD), a strong predictor for AKI, was identified using $\mathrm{PCr}$ measurements from the LABKA database. We defined CKD as an estimated glomerular filtration rate $<60 \mathrm{~mL} / \mathrm{min} / 1.73 \mathrm{~m}^{2}$ lasting at least 3 months within the 2 years before CRC surgery. ${ }^{19}$

The following covariates were identified from the DNPR, based on an inpatient or outpatient hospital contact for a given condition within 10 years before CRC surgery: obstructive pulmonary disease, arterial hypertension, diabetes mellitus, heart disease and liver disease. To improve the sensitivity of diabetes mellitus and obstructive pulmonary disease diagnoses, we searched the National Health Service Prescription Database (NHSPD) for previous prescriptions of medications used to treat these conditions. ${ }^{15}$ The NHSPD contains records for all reimbursable drugs dispensed in community pharmacies in Denmark since 2004. Body mass index (BMI) was computed from weight and height data retrieved from the DCCG database, ${ }^{12}$ divided into three categories: underweight $\left(<18.5 \mathrm{~kg} / \mathrm{m}^{2}\right)$, normal weight $\left(18.5-24.9 \mathrm{~kg} / \mathrm{m}^{2}\right)$ and overweight $\left(\geq 25 \mathrm{~kg} / \mathrm{m}^{2}\right){ }^{20}$ 
Patients undergoing CRC surgery in Northern Denmark during 2005-2011

$(n=6,768)$

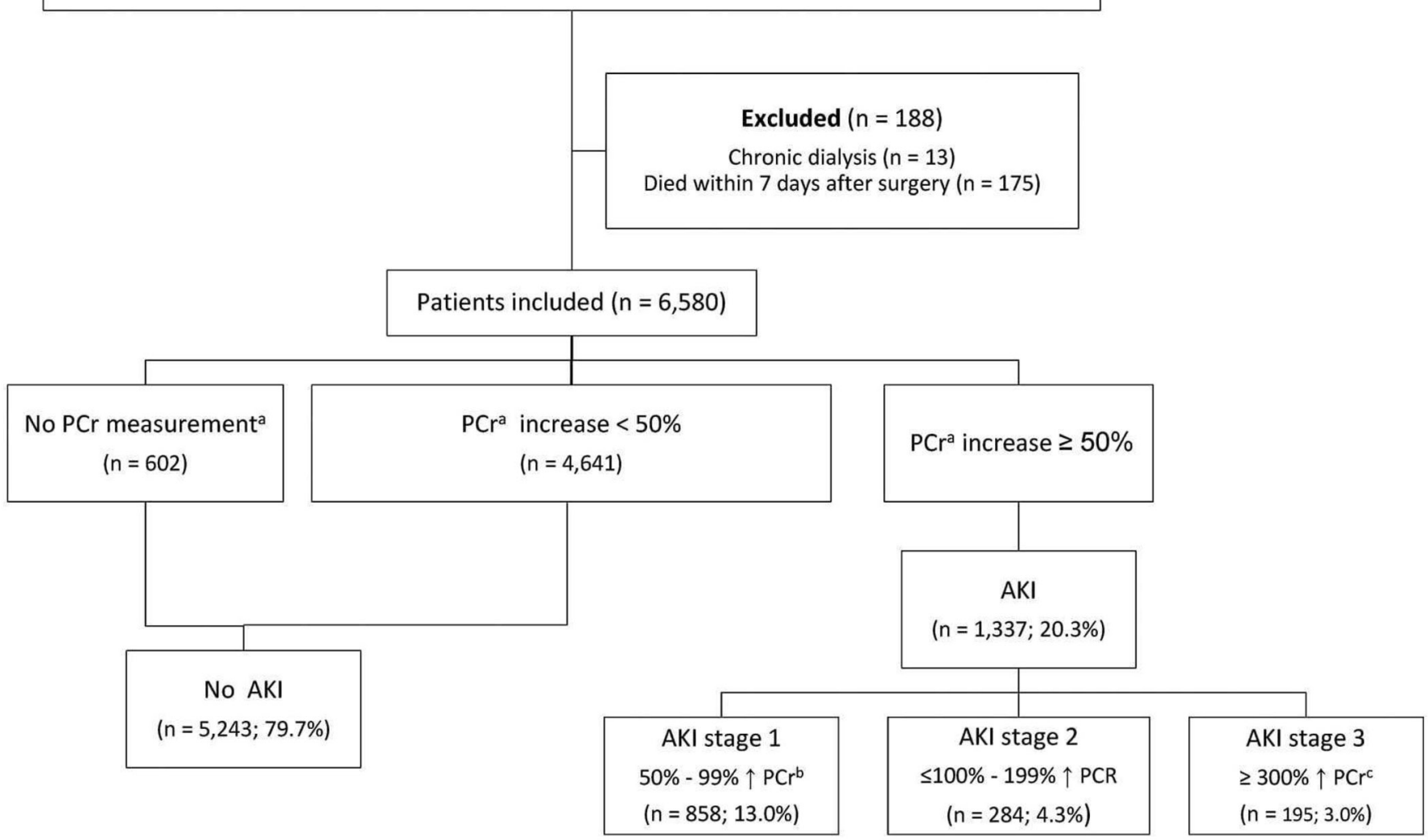

${ }^{a}$ Within seven days after surgery.

b Or a $26,5 \mu \mathrm{mol} / \mathrm{l}$ increase within two days after surgery.

${ }^{\text {c }}$ Or a $\mathrm{PCr}$ above $353.6 \mu \mathrm{mol} / \mathrm{l}$, with an acute increase of at least $44 \mu \mathrm{mol} / \mathrm{I}$ or with acute administration of dialysis.

Figure 1 Flow chart and AKI definition. AKI, acute kidney injury; CRC, colorectal cancer; PCr, plasma creatinine.

\section{Statistical methods}

Patient characteristics, including demographics, comorbidities and information from the hospitalisation that included CRC surgery, were tabulated by AKI stage (table 1).

Because we assessed AKI within the first 7 days after surgery, we followed patients from day 8 after surgery until death, emigration or up to 1 year after surgery, whichever came first. The Kaplan-Meier method was used to compute cumulative mortality curves (1-survival function) for patients with and without AKI. ${ }^{21}$ AKI was further disaggregated into AKI stages $1-3 .{ }^{8}$ We computed HRs of death within 8-30, 31-90 and 91-365 days after surgery, comparing patients with AKI (and for each stage of AKI) with patients without AKI, using a Cox proportional hazards regression model adjusted for potential confounders. Confounders included age group (0-49, $50-59,60-69,70-79, \geq 80$ ), gender, BMI category, CKD (without CKD, stage 3 or stage 4 and higher), diabetes, obstructive pulmonary disease, hypertension, liver disease, heart disease, tumour site (colon or rectum) and urgency of surgery (acute or elective). The assumption of proportional hazards was checked graphically and found appropriate within each of the follow-up periods 8-30, 31-90 and
91-365 days after surgery. To address potential different effects in subgroups (effect modification), we repeated the analyses stratified by sex, age, CKD stage, acute versus elective surgery, surgical approach (open or minimally invasive), type of surgical procedure, diabetes mellitus and year of surgery. Moreover, a sensitivity analysis including follow-up from 0 to 30 days after surgery was performed.

\section{Missing data}

Information on lifestyle variables (BMI, smoking and alcohol use) was missing for approximately $30 \%$ of patients (table 1) and $20 \%$ for CKD. We did not have exact date of anastomosis leakage and other surgical complications occurring within 30 days after surgery and were therefore not able to assess the temporal relationship.

We used two approaches to address potential misclassification stemming from missing data. First, we conducted a complete-case analysis. Second, in a sensitivity analysis, we performed multiple imputations, calculating average HRs for 30 imputed datasets. In the sensitivity analysis, we estimated values for missing data for five categorical variables (CKD, urgency of surgery, BMI, smoking and alcohol intake) using all covariates, the outcome and the 
Table 1 Patient characteristics by AKI stage, Denmark, 2005-2011

\begin{tabular}{|c|c|c|c|c|c|c|}
\hline \multirow[b]{3}{*}{ Patient characteristics } & \multirow[b]{2}{*}{ Without AKI } & \multirow[b]{2}{*}{ AKI } & \multicolumn{3}{|l|}{ AKI stage } & \multirow[b]{2}{*}{ All patients } \\
\hline & & & Stage 1 & Stage 2 & Stage 3 & \\
\hline & No (\%) & No (\%) & No (\%) & No (\%) & No (\%) & No (\%) \\
\hline Total (row \%) & $5243(79.7)$ & $1337(20.3)$ & 858 (13.0) & $284(4.3)$ & 195 (3.0) & $6580(100)$ \\
\hline $\begin{array}{l}\text { Mean duration of AKI } \\
\text { (days) }\end{array}$ & - & 2.1 & 1.4 & 2.5 & 4.3 & - \\
\hline \multicolumn{7}{|l|}{ Sex } \\
\hline Female & 2565 (48.9) & 513 (38.4) & 329 (38.3) & $111(39.1)$ & 73 (37.4) & 3078 (46.8) \\
\hline Male & 2678 (51.1) & 824 (61.6) & $529(61.7)$ & $173(60.9)$ & $122(62.6)$ & 3502 (53.2) \\
\hline \multicolumn{7}{|l|}{ Age (years) } \\
\hline $0-49$ & 277 (5.3) & $40(3.0)$ & $21(2.4)$ & $8(2.8)$ & $11(5.6)$ & $317(4.8)$ \\
\hline $50-59$ & 753 (14.4) & $130(9.7)$ & $82(9.6)$ & 35 (12.3) & $13(6.7)$ & $883(13.4)$ \\
\hline $60-69$ & 1555 (29.7) & 323 (24.2) & $196(22.8)$ & $74(26.1)$ & $53(27.2)$ & $1878(28.5)$ \\
\hline $70-79$ & 1643 (31.3) & 497 (37.2) & $313(36.5)$ & 103 (36.3) & $81(41.5)$ & 2140 (32.5) \\
\hline$\geq 80$ & 1015 (19.4) & 347 (26.0) & $246(28.7)$ & $64(22.5)$ & 37 (19.0) & $1362(20.7)$ \\
\hline \multicolumn{7}{|c|}{$\mathrm{BMI}$} \\
\hline Normal & 1755 (33.5) & 375 (28.0) & 245 (28.6) & 83 (29.2) & $47(24.1)$ & $2130(32.4)$ \\
\hline Underweight & $124(2.4)$ & $26(1.9)$ & $22(2.6)$ & $3(1.1)$ & $1(0.5)$ & $150(2.3)$ \\
\hline Overweight & 1835 (35.0) & 512 (38.3) & 317 (36.9) & $111(39.1)$ & $84(43.1)$ & 2347 (35.7) \\
\hline Missing & 1529 (29.2) & $424(31.7)$ & 274 (31.9) & 87 (30.6) & $63(32.3)$ & $1953(29.7)$ \\
\hline Heart disease & $67(1.3)$ & $23(1.7)$ & $16(1.9)$ & $5(1.8)$ & $2(1.0)$ & $90(1.4)$ \\
\hline Diabetes mellitus & $479(9.1)$ & 185 (13.8) & 115 (13.4) & $43(15.1)$ & 27 (13.8) & $664(10.1)$ \\
\hline Liver disease & $50(1.0)$ & $19(1.4)$ & $10(1.2)$ & $6(2.1)$ & $3(1.5)$ & $69(1.0)$ \\
\hline Arterial hypertension & $1260(24)$ & 479 (35.8) & $304(35.4)$ & 95 (33.5) & $80(41.0)$ & $1739(26.4)$ \\
\hline OPD & $512(9.8)$ & 166 (12.4) & $110(12.8)$ & $32(11.3)$ & $24(12.3)$ & $678(10.3)$ \\
\hline \multicolumn{7}{|l|}{ CKD } \\
\hline Stage 3 & 834 (15.9) & 347 (26.0) & 251 (29.3) & $59(20.8)$ & 37 (19.0) & $1181(17.9)$ \\
\hline Stage 4 or higher & $39(0.7)$ & $48(3.6)$ & $25(2.9)$ & $2(0.7)$ & $21(10.8)$ & $87(1.3)$ \\
\hline \multicolumn{7}{|l|}{ Cancer type } \\
\hline Colon & $3481(66.4)$ & $862(64.5)$ & 540 (62.9) & $186(65.5)$ & $136(69.7)$ & $4343(66.0)$ \\
\hline Rectum & $1762(33.6)$ & 475 (35.5) & $318(37.1)$ & $98(34.5)$ & 59 (30.3) & 2237 (34.0) \\
\hline \multicolumn{7}{|l|}{ Urgency of surgery } \\
\hline Elective & 4540 (86.6) & $1102(82.4)$ & $713(83.1)$ & $232(81.7)$ & $157(80.5)$ & $5642(85.7)$ \\
\hline Acute & $542(10.3)$ & 208 (15.6) & $129(15.0)$ & $47(16.5)$ & 32 (16.4) & 750 (11.4) \\
\hline Missing & $161(3.1)$ & $27(2.0)$ & $16(1.9)$ & $5(1.8)$ & $6(3.1)$ & $188(2.9)$ \\
\hline \multicolumn{7}{|l|}{ UICC stage } \\
\hline Stage I & $773(14.7)$ & $181(13.5)$ & $116(13.5)$ & $38(13.4)$ & 27 (13.8) & $954(14.5)$ \\
\hline Stage II & 1899 (36.2) & 515 (38.5) & 329 (38.3) & 99 (34.9) & $87(44.6)$ & 2414 (36.7) \\
\hline Stage III & $1412(26.9)$ & 387 (28.9) & $247(28.8)$ & 97 (34.2) & $43(22.1)$ & $1799(27.3)$ \\
\hline Stage IV & 904 (17.2) & 217 (16.2) & 140 (16.3) & $44(15.5)$ & 33 (16.9) & $1121(17)$ \\
\hline Missing & $255(4.9)$ & $37(2.8)$ & $26(3)$ & $6(2.1)$ & $5(2.6)$ & $292(4.4)$ \\
\hline Mechanical ventilation* & $35(0.7)$ & 155 (11.6) & $53(6.2)$ & $39(13.7)$ & 63 (32.3) & $190(2.9)$ \\
\hline Vasopressor/inotropes* & $54(1.0)$ & $163(12.2)$ & $56(6.5)$ & $38(13.4)$ & $69(35.4)$ & 217 (3.3) \\
\hline Preoperative* radiation & $564(10.8)$ & $142(10.6)$ & $94(11)$ & 33 (11.6) & $15(7.7)$ & $706(10.7)$ \\
\hline Preoperative† chemo & $3(0.1)$ & $1(0.1)$ & $1(0.1)$ & $0(0)$ & $0(0)$ & $4(0.1)$ \\
\hline
\end{tabular}




\begin{tabular}{|c|c|c|c|c|c|c|}
\hline \multirow[b]{3}{*}{ Patient characteristics } & \multirow[b]{2}{*}{ Without AKI } & \multirow[b]{2}{*}{ AKI } & \multicolumn{3}{|l|}{ AKI stage } & \multirow[b]{2}{*}{ All patients } \\
\hline & & & Stage 1 & Stage 2 & Stage 3 & \\
\hline & No (\%) & No (\%) & No (\%) & No $(\%)$ & No (\%) & No $(\%)$ \\
\hline $\begin{array}{l}\text { Preadmission } \ddagger \text { NSAID } \\
\text { use }\end{array}$ & $532(10.1)$ & $150(11.2)$ & $93(10.8)$ & $36(12.7)$ & $21(10.8)$ & $682(10.4)$ \\
\hline Preadmission $\ddagger$ ACE use & $738(14.1)$ & $266(19.8)$ & $156(18.2)$ & $61(21.5)$ & $49(25.1)$ & $1004(15.3)$ \\
\hline
\end{tabular}

*Treatment received during the CRC surgery admission.

†Defined as radiation/chemotherapy within 3 months before surgery.

$\ddagger$ Defined as one or more redeemed prescriptions within 90 days before the surgery.

AKI, acute kidney injury; ASA, American Society of Anesthesiologists; BMI, body mass index; CRC, colorectal cancer; CKD, chronic kidney disease; NSAID, non-steroidal anti-inflammatory drugs; OPD, obstructive pulmonary disease; UICC, Union for International Cancer Control.

Nelson-Aalen estimator of the cumulative baseline hazard to observed survival time. ${ }^{22}$ We expected that data were missing at random, but not completely at random, in our study because younger and healthier patients were more likely to have missing data.

\section{Timing of AKI}

We addressed timing of AKI and other complications as follows. For all patients who developed AKI within 7 days after CRC surgery, we cross-tabulated the first day of AKI with typical late complications (occurring 6-8 days postsurgery), for example, anastomosis leakage and fascial dehiscence (figure 2).

All analyses were conducted using the Stata software package, V.13.1 (StataCorp). All data were obtained from Danish registries and, in accordance with Danish law, their use did not require ethical approval or informed consent.

\section{RESULTS}

\section{Study population and AKI occurrence}

We identified 6768 patients with CRC residing in Northern Denmark who underwent CRC surgery during 1 January

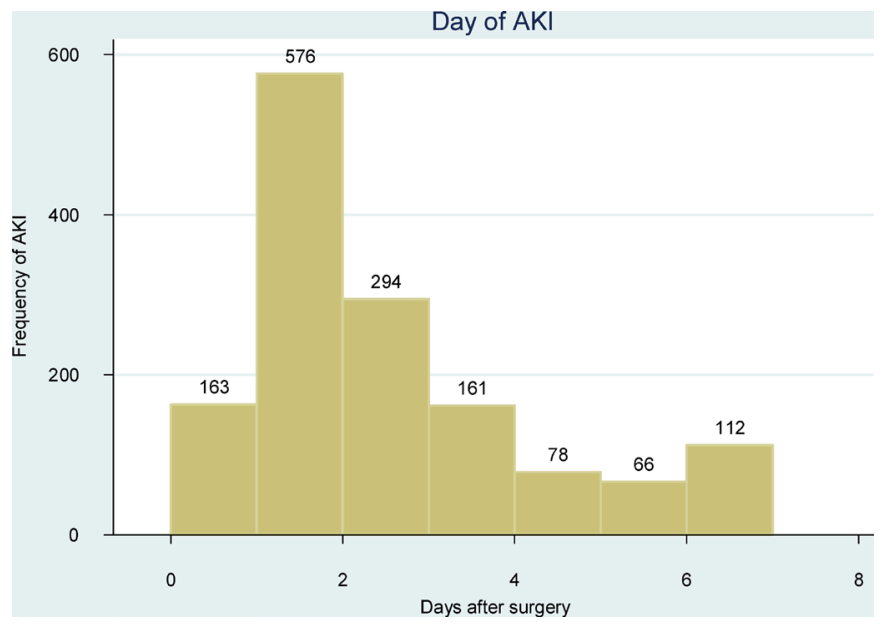

Figure 2 Number of patients who developed acute kidney injury, by day after surgery for all patients. No late complications $=$ fascial dehiscence, ileus, anastomotic leakage. AKI, acute kidney injury.
2005 to 31 December 2011. Of these, 188 patients were excluded due to either chronic dialysis $(n=13)$ and death before start of follow-up (8 days after surgery) $(n=175)$. Of the 175 patients dying within 7 days after surgery, 113 (64.6\%) had AKI and $62(35.4 \%)$ did not have AKI. In total, 6580 patients were included in the analyses. Postoperative AKI within 7 days after surgery occurred in 1337 patients (20.3\%). AKI stage was distributed as follows among study patients: 858 had stage 1 AKI (13.0\%), 284 had stage 2 AKI $(4.3 \%)$ and 195 had stage 3 AKI (3.0\%) (figure 1). Among patients with stage 3 AKI, 67 (34.4\%) received RRT. Moreover, AKI duration seemed to be more likely to be longer in patients with increasing severity of AKI.

\section{Descriptive data}

Total follow-up time was 5741 person-years and there was no lost to follow-up. Median age was 71.3 years, 2678 (53.3\%) patients were male and $3481(66.0 \%)$ had colon cancer (table 1).

Compared with patients without AKI, those with AKI were more commonly men, aged 70 years or more, with a history of smoking and a BMI categorised as overweight (table 1). Patients with AKI also had a higher American Society of Anesthesiologists' Classification of Physical Health score. ${ }^{23}$ Thus, patients with AKI more often had preoperative CKD, diabetes mellitus, arterial hypertension or were treated with ACE-inhibitors than patients without AKI. Moreover, postoperatively, patients with AKI more often received mechanical ventilation were treated with vasopressors/inotropes, or developed postoperative bleeding, ileus, fascial dehiscence and infectious complications than patients without AKI. The prevalence rate ratio of anastomosis leakage in patients with AKI compared with patients without AKI was 3.71 (95\% CI 3.03 to 4.53 ) (online supplementary table S1).

\section{Mortality}

Cumulative 8-30 days mortality was higher in patients with AKI $(10.1 \%)$ than in patients without AKI (table 2). Compared with patients without AKI, the adjusted HR (aHR) for patients with AKI was 4.01 (95\% CI 3.11 to 5.17). Mortality increased by AKI stage and was higher for patients with stage 1 AKI than for patients without AKI (table 2 and figure 3 ). 
Table 2 Mortality outcomes (8-30, 31-90 and 91-365 days mortality) by AKI

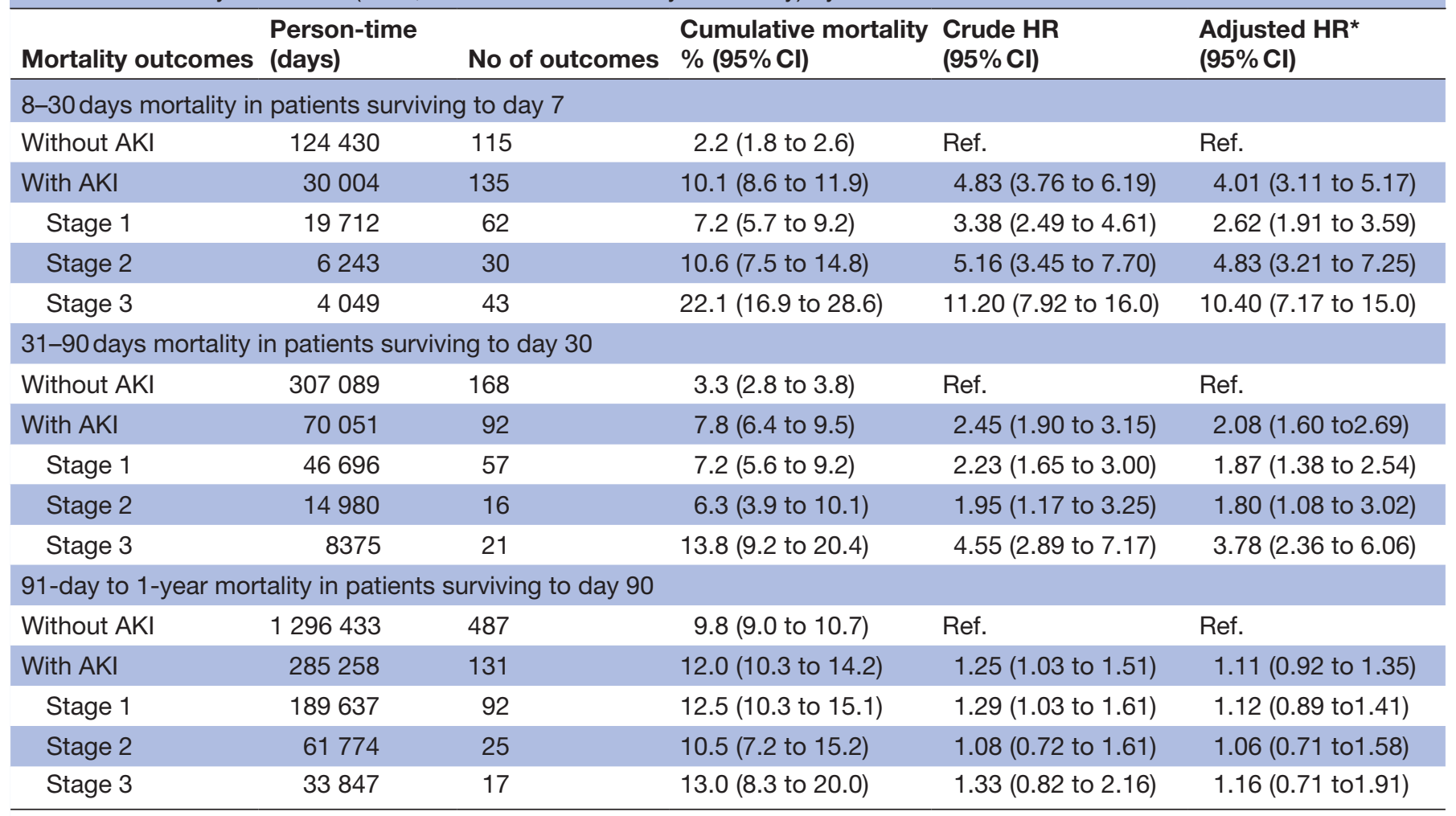

*Adjusted for age (categories: 0-49, 50-59, 60-69, 70-79 and $\geq 80$ years), gender, BMI category, CKD stages (3-5), diabetes mellitus (yes/no), obstructive pulmonary disease, hypertension, liver disease, heart disease, tumour site and acute versus elective surgery.

$\mathrm{AKI}$, acute kidney injury; BMI, body mass index; CKD, chronic kidney disease.

For patients who survived the first 30 days after CRC surgery, cumulative mortality during the following 31-90 days was higher in patients with AKI $(7.8 \%)$ than in patients without AKI (3.3\%) (table 2). The aHR for mortality was 2.08 (95\% CI 1.60 to 2.69$)$ in patients with AKI compared with patients without AKI. Mortality also appeared to increase by AKI stage (table 2 and figure 3 ).

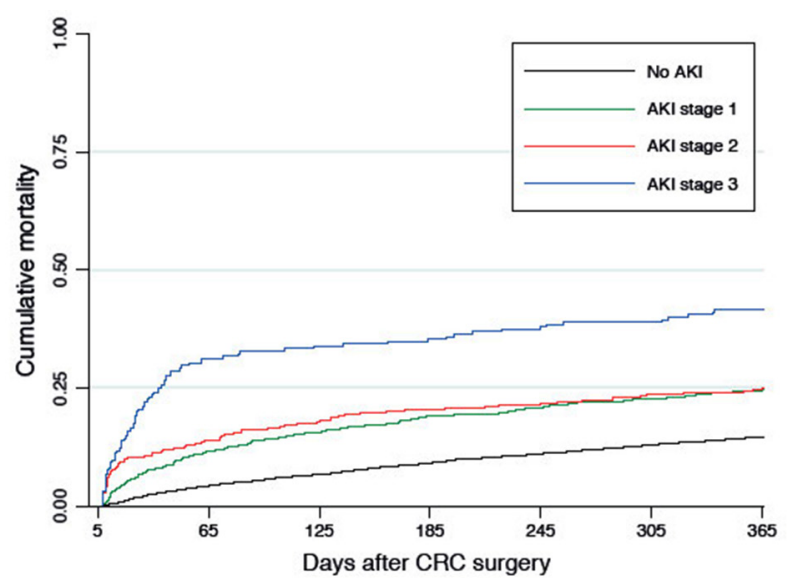

Figure 3 Cumulative 1-year mortality by acute kidney injury stage within 7 days after colorectal cancer surgery, Northern Denmark, January 2005-December 2011. AKI, acute kidney injury; CRC, colorectal cancer.
Cumulative 91-365 days mortality following surgery initially appeared higher in patients with AKI compared with patients without AKI: $12.0 \%$ vs $9.8 \%$ (table 2 and figure 3). However, the association attenuated after adjustment (aHR=1.11, 95\% CI 0.92 to 1.35$)$.

\section{Sensitivity analyses}

Sensitivity analyses performed to address missing data, including both multiple imputation and a completecase analysis, yielded no discernible change in mortality (online supplementary tables S2 and S3). A sensitivity analysis including follow-up from 0 to 30 days after surgery was performed and showed no noticeable differences in estimates as compared with follow-up from day 8 (online supplementary table S4).

\section{Subgroup analyses}

While we observed no major differences between subgroups, our analyses yielded aHRs with very wide CIs, making the point estimates less reliable and precluding conclusions about the presence of effect modification (online supplementary figure S1).

\section{Timing of AKI and late complications after surgery}

Overall, as well as in patients with late complications such as anastomosis leakage or fascial dehiscence, AKI occurred most frequently within 1-4 days after surgery. 


\section{DISCUSSION}

\section{Key results}

In this population-based cohort study, we found that $20 \%$ of patients developed AKI within 7 days following CRC surgery. AKI was associated with a twofold to threefold higher risk of mortality within the first 90 days after CRC surgery even in patients with stage 1 AKI. In the period 91-365 days after surgery, mortality was similar in patients with and without AKI, after adjustment. During the first 8-30 days after surgery, the association between postoperative AKI and mortality appeared to increase with higher AKI stage. In general, patients with AKI had more postoperative complications.

\section{Previous studies}

Two studies reported on kidney dysfunction and mortality in postoperative patients with CRC. ${ }^{10}{ }^{11}$ Iversen et al examined postoperative complications and mortality in 2157 Danish patients with CRC who underwent emergency surgery. ${ }^{10}$ However, their study included only patients with AKI receiving RRT. While AKI was reported by surgeons to the DCCG database within 30 days, the severity of AKI in patients receiving RRT reduced the risk of under-reporting. Iversen $e t$ al found that $2.0 \%$ of postoperative patients with CRC had AKI and were receiving RRT and 30 -day mortality among these patients was $68 \%$. This is comparable with our results for patients with stage 3 AKI who received RRT. ${ }^{10}$ The other study did investigate occurrence of all AKI stages after rectal cancer resections, although mortality (in-hospital) was only reported for overall AKI $(18.2 \%){ }^{11}$

Our results also are consistent with reported prevalences of 3\%-35\% for postoperative AKI and $0.5 \%-25 \%$ rates of all-cause postoperative mortality within the first year in patients who underwent major abdominal surgery. ${ }^{7}$

\section{Strengths and limitations}

The risk of selection bias in our study was limited for three reasons. First, patients were included in relevant databases as part of the prospective registration of data for administrative purposes (DNPR, CRS, LABKA and NHSPD) or to evaluate clinical quality (DCCG) in Danish hospitals. Second, study participants had uniform access to healthcare with complete follow-up. Third, the completeness of the databases $(85 \%-99 \%) \%)$ and of most variables were high. ${ }^{12-16}$

Supported by the results of our sensitivity analyses, we conclude that misclassification due to inclusion of patients lacking exposure information in the group without AKI (11\%) posed a minor risk of information bias.

The KDIGO criteria include urine output. As in many other studies in non-intensive care unit settings, AKI staging for our study was performed without information on urine output, which was unavailable. Consequently, the lack of data on urine output may have led to misclassification that could have biased our results towards the null. We expect that the bias is limited since $\mathrm{SCr}$ alone has a stronger association with mortality than urine output. ${ }^{2324}$
In our study, AKI may have been related to other surgical complications and accompanying reoperations. Unfortunately, the date of postoperative complications is unavailable in the DCCG database. Therefore, we could not assess the timing of AKI and development of other postoperative complications that could potentially lead to AKI. Also, our study was limited by the lack of information on type of anaesthesia, perioperative fluid administration and contrast exposure.

As well, information on BMI (self-reported at hospital admission) was missing for $30 \%$ of patients. A multiple imputation analysis addressing this missing information yielded only minor changes in our results. Despite this analysis and extensive adjustment for potential confounders including lifestyle variables, we cannot entirely rule out unmeasured confounding from such factors as fluid management, for which we had no information, and residual confounding. Still, our study clearly showed that AKI is a marker for increased risk of death after CRC surgery.

\section{Interpretation}

AKI is a multifactorial condition, and in the operative setting, relevant factors include response to anaesthesia (eg, peripheral vasodilatation and myocardial depression) and surgery (increase in the antidiuretic hormone and aldosterone), as well as the effect of fluid depletion. Major surgery introduces the risk of fluid depletion at several stages, for example, preoperative nil-by-mouth regimen, perioperative blood and intravascular fluid loss, extravasation of fluid from the vascular compartment (third-space effect), insensible fluid losses and the pathology of the disease itself.

AKI also could develop from complications such as sepsis or electrolyte derangement associated with ileus. This could be due to global hypoperfusion of the kidney if the afferent arteriole dilation and efferent arteriole vasoconstriction response initiated by the kidney does not result in adequate glomerular filtration. Further, hypotension can lead to dysfunctional intrarenal microcirculation due to patchy areas of hypoperfusion in the kidney and potentially add to the risk of developing AKI. ${ }^{25-28}$

AKI most frequently occurred during the initial days following surgery. In patients with late complications (ileus, fascial dehiscence or anastomotic leakage), there was an additional peak after 5-6 days, corresponding to reoperations for these complications. AKI might also contribute to the development of such postoperative complications. This hypothesis needs to be investigated further in studies in which the timing of AKI and postoperative complications can be ascertained.

The results of the study are generalisable to other CRC surgery settings with a population resembling the Danish population despite our restriction to North Denmark Region and Central Denmark Region, because the Danish Healthcare system is homogeneous across regions, hospitals, coding practice, socioeconomic characteristics and healthcare usage. ${ }^{29}$ 
In conclusion, AKI occurred in approximately $20 \%$ of patients undergoing CRC surgery and was associated with increased mortality throughout the first 90 days following surgery, even in patients with stage 1 AKI (ie, with more than a $50 \%$ increase in creatinine). Thus, patients with even small increases in creatinine may require enhanced attention to achieve early detection of AKI and correction of fluid and electrolyte derangement. An interventional study is required to examine whether such interventions will reduce the rate or at least the severity of postoperative complications.

Acknowledgements We thank Biostatisticians Thomas Bøjer Rasmussen and Uffe Heide-Jørgensen, Department of Clinical Epidemiology, Aarhus University Hospital, Aarhus, Denmark, for statistical advice regarding data management and analyses.

Contributors CS: Protocol, data retrieval and management, analyses, manuscript. HG: Protocol, assistance with data management and analyses, major revision of the manuscript. LHI: Discussion and choice of inclusion/exclusion criteria for patients, based on extensive knowledge of the Danish Colorectal Cancer Group database and clinical skills, major revision of the manuscript. HTS: Protocol, discussion and choice of analyses, major revision of the manuscript. CFC: Protocol, discussion and choice of analyses, major revision of the manuscript.

Funding The study was funded by the 'Linexfonden', by the Health Research Fund of Central Denmark Region and by the Program for Clinical Research Infrastructure (PROCRIN).

Disclaimer These entities had no role in the design of the study, collection, analysis, interpretation of the data or in writing the manuscript.

Competing interests None declared.

Patient consent for publication Not required.

Ethics approval The study was approved by the Danish Data Protection Agency (record no. 2015-57-0002, Aarhus University record no. 2016-051-000001/423).

Provenance and peer review Not commissioned; externally peer reviewed.

Data sharing statement The data, analytical methods and study materials will not be made available to other researchers for purposes of reproducing the results or replicating the procedure. Such disclosure would conflict with the regulations for use of Danish healthcare data.

Open access This is an open access article distributed in accordance with the Creative Commons Attribution Non Commercial (CC BY-NC 4.0) license, which permits others to distribute, remix, adapt, build upon this work non-commercially, and license their derivative works on different terms, provided the original work is properly cited, appropriate credit is given, any changes made indicated, and the use is non-commercial. See: http://creativecommons.org/licenses/by-nc/4.0/.

\section{REFERENCES}

1. Bosman FT, Hamilton SR R. World cancer report: World Health Organization, 2014.

2. Spanjersberg WR, Reurings J, Keus F, et al. Fast track surgery versus conventional recovery strategies for colorectal surgery. Cochrane Database Syst Rev 2011:CD007635.

3. Greco M, Capretti G, Beretta L, et al. Enhanced recovery program in colorectal surgery: a meta-analysis of randomized controlled trials. World J Surg 2014;38:1531-41.

4. Iversen LH, Ingeholm P, Gögenur I, et al. Major reduction in 30-day mortality after elective colorectal cancer surgery: a nationwide population-based study in Denmark 2001-2011. Ann Surg Oncol 2014;21:2267-73.
5. Morris EJ, Taylor EF, Thomas JD, et al. Thirty-day postoperative mortality after colorectal cancer surgery in England. Gut 2011;60:806-13.

6. Gameiro J, Neves JB, Rodrigues N, et al. Acute kidney injury, long-term renal function and mortality in patients undergoing major abdominal surgery: a cohort analysis. Clin Kidney J 2016;9:192-200.

7. Gameiro J, Fonseca JA, Neves M, et al. Acute kidney injury in major abdominal surgery: incidence, risk factors, pathogenesis and outcomes. Ann Intensive Care 2018;8:22

8. KDIGO. Kidney Disease: Improving Global Outcomes (KDIGO) Acute kidney injury work group. 2: Kidney International Supplements, 2012.

9. Bellomo R, Ronco C, Kellum JA, et al. Acute renal failure - definition, outcome measures, animal models, fluid therapy and information technology needs: the Second International Consensus Conference of the Acute Dialysis Quality Initiative (ADQI) Group. Crit Care 2004;8:R204-9.

10. Iversen LH, Bülow S, Christensen IJ, et al. Postoperative medical complications are the main cause of early death after emergency surgery for colonic cancer. Br J Surg 2008;95:1012-9.

11. Lim SY, Lee JY, Yang JH, et al. Predictive factors of acute kidney injury in patients undergoing rectal surgery. Kidney Res Clin Pract 2016;35:160-4.

12. Grann AF, Erichsen R, Nielsen AG, et al. Existing data sources for clinical epidemiology: the clinical laboratory information system (LABKA) research database at Aarhus University, Denmark. Clin Epidemiol 2011;3:133-8.

13. Schmidt M, Schmidt SA, Sandegaard JL, et al. The danish national patient registry: a review of content, data quality, and research potential. Clin Epidemiol 2015;7:449-90.

14. Schmidt M, Pedersen L, Sørensen HT. The danish civil registration system as a tool in epidemiology. Eur J Epidemiol 2014;29:541-9.

15. Johannesdottir SA, Horváth-Puhó E, Ehrenstein V, et al. Existing data sources for clinical epidemiology: the danish national database of reimbursed prescriptions. Clin Epidemiol 2012;4:303-13.

16. Ingeholm P, Gögenur I, Iversen LH. Danish colorectal cancer group database. Clin Epidemiol 2016;8:465-8.

17. Miles RR, Roberts RF, Putnam AR, et al. Comparison of serum and heparinized plasma samples for measurement of chemistry analytes. Clin Chem 2004;50:1704-6.

18. Kheterpal S, Tremper KK, Englesbe MJ, et al. Predictors of postoperative acute renal failure after noncardiac surgery in patients with previously normal renal function. Anesthesiology 2007;107:892-902

19. Kdigo. K. Clinical practice guideline for the evaluation and management of chronic kidney disease: Kidney International Supplements, 2013:3.

20. Europe WHO, undefined. Body mass index (BMI): World Health Organization Europe, 2018.

21. Kaplan EL P. Nonparametric estimation from incomplete observations. , 1958:53, 457-81.

22. Sterne JA, White IR, Carlin JB, et al. Multiple imputation for missing data in epidemiological and clinical research: potential and pitfalls. BMJ 2009;338:b2393.

23. Morgan DJ, Ho KM. A comparison of nonoliguric and oliguric severe acute kidney injury according to the risk injury failure loss end-stage (RIFLE) criteria. Nephron Clin Pract 2010;115:c59-65.

24. Panagiotou A, Garzotto F, Gramaticopolo S, et al. Continuous real-time urine output monitoring for early detection of acute kidney injury. Contrib Nephrol 2011;171:194-200.

25. Ostermann M, Liu K. Pathophysiology of AKI. Best Pract Res Clin Anaesthesiol 2017;31:305-14.

26. Calvert S, Shaw A. Perioperative acute kidney injury. Perioper Med 2012;1:1-11.

27. Sear JW. Kidney dysfunction in the postoperative period. $\mathrm{Br} \mathrm{J}$ Anaesth 2005;95:20-32.

28. Carmichael P, Carmichael AR. Acute renal failure in the surgical setting. ANZ J Surg 2003;73:144-53.

29. Henriksen DP, Rasmussen L, Hansen MR, et al. Comparison of the five danish regions regarding demographic characteristics, healthcare utilization, and medication use--a descriptive crosssectional study. PLoS One 2015;10:e0140197. 